\title{
Synthesis and binding site characteristics of 2,4,6-trichlorophenol-imprinted polymers
}

\author{
Qin-Zhong Feng • Li-Xia Zhao • Bao-Lin Chu • \\ Wei Yan · Jin-Ming Lin
}

Received: 2 July 2008 /Revised: 1 September 2008 /Accepted: 4 September 2008 / Published online: 27 September 2008

(C) Springer-Verlag 2008

\begin{abstract}
Trichlorophenol (2,4,6-TCP)-imprinted micro- and submicrospheres prepared by precipitation polymerization were compared with templated materials obtained by conventional bulk polymerization. The influence of the type and amount of functional monomer, the type and amount of cross-linker, polymerization temperature, porogen, and the ratio of template molecule and functional monomer to cross-linker on the size of the obtained particles were investigated. UV-Vis spectrophotometer experiments revealed that the microsphere polymers provided higher affinity to the template in contrast to imprinted polymers prepared by bulk polymerization. The binding properties of the microspheres, including binding isotherms and affinity distribution, were studied via Freundlich isotherm affinity distribution (FIAD) analysis. The obtained results indicated that microspheres prepared by precipitation polymerization provided superior rebinding properties during equilibrium binding in contrast to bulk polymers and submicrosphere polymers. Moreover, release experiments showed that $80 \%$ of rebound $2,4,6-\mathrm{TCP}$ was released from the imprinted microspheres within the first $2 \mathrm{~h}$, while more intimately bound 2,4,6-TCP molecules were released in the following $40 \mathrm{~h}$. The morphologies and porosities of the resulting imprinted materials were charac-
\end{abstract}

Q.-Z. Feng $\cdot$ L.-X. Zhao $(\bowtie) \cdot$ W. Yan · J.-M. Lin $(\bowtie)$

State Key Laboratory of Environmental Chemistry and

Ecotoxicology, Research Center for Eco-Environmental Sciences,

Chinese Academy of Sciences,

P. O. Box 2871, Beijing 100085, China

e-mail: zlx@rcees.ac.cn

e-mail: jmlin@mail.rcees.ac.cn

B.-L. Chu

State Key Laboratory of Chemical Resource Engineering,

Beijing University of Chemical Technology,

Beijing 100029, China terized by scanning electron microscopy (SEM) and Brunauer-Emmett-Teller (BET) analysis, respectively. The microsphere polymers exhibited a regular spherical shape with a high degree of monodispersity to the corresponding bulk polymers. Furthermore, the micro- and submicrospheres were characterized by narrow distribution of pores in contrast to a heterogeneity index of $m=0.6647$ for the microsphere imprinted polymer.

Keywords Molecularly imprinted polymers .

Precipitation polymerization - Imprinted microsphere .

Imprinted submicrosphere $\cdot 2,4,6$-Trichlorophenol

\section{Introduction}

Molecularly imprinted polymers (MIPs) can be readily tailored with selectivity for a guest molecule. This is accomplished by synthesizing a network polymer in the presence of a template molecule. Removal of the template from the polymeric matrix leaves cavities of complementary size, shape, and chemical functionality to the template $[1,2]$. MIPs offer some advantages, for example, they are stable to a wide range of pressures, organic solvents, and pHs. These properties make MIPs attractive for many analytical applications as stationary phases for chromatographic separations $[3,4]$, for molecular and ionic separations by selective sorption $[5,6]$, as recognition entities in sensors $[7,8]$, in immunoassays [9, 10], and as catalysts [11, 12].

By far the most widely used technique for the preparation of such materials gives particles of irregular shape through a low-yield, time-consuming crush-and-sieve process [13]. Although these particles often exhibit high affinity and selectivity for the target in question, the wide use of these materials has not been realized. This is 
primarily because of low capacity and poor site accessibility for the target molecule [14]. Moreover, the preparation procedures are time- and labor-consuming and the prepared particles often have wide size distributions. These problems have been addressed by using bead polymerization techniques [15], either in two-phase systems by suspension polymerization $[16,17]$ or in one-phase systems by precipitation polymerization [18]. Alternatively, beaded MIPs may be obtained via the use of grafting procedures where one distinguishes between "grafting to" and "grafting from" techniques [19], the latter referring to polymerization initiated from a preformed polymer or from the surface of a solid support. However, these techniques suffer from the presence of water or highly polar organic solvents during the polymerization procedure, which frequently decreases specific interactions between functional monomers and template molecules in most commonly applied noncovalent imprinting mixtures [16]. Hence, for the synthesis of molecularly imprinted micro- and nanospheres retaining high selectivity along with control of particle shape and size, a synthetic route avoiding the use of dispersants such as water or polar solvents is demanded. Precipitation techniques have been applied for the preparation of imprinted nanospheres for a variety of different analytes and applications [20,21]. Most obtained spherical particles have diameters ranging from 0.1 to $1 \mu \mathrm{m}$ with the diameter dispersivity usually narrowed by centrifugation. The dimensions and morphologies of these spheres are ideal for radioligand binding assays $[20,21]$ and capillary electrophoresis [20]; however, they are less suitable for HPLC and SPE applications due to the resulting high backpressure of columns packed with such small particles. For example, imprinted microspheres for $17 \beta$-estradiol with a diameter less than $1 \mu \mathrm{m}$ have been developed by Mosbach's group [20, 21] for radioligand binding assays based on trimethylolpropane trimethacrylate (TRIM, as cross-linker) and acetonitrile (as porogen). Hence, the synthesis of larger particles by simple precipitation polymerization yielding monodisperse MIP-based stationary phase materials requires the development of a novel synthetic route.

2,4,6-Trichlorophenol (2,4,6-TCP) is used as an antiseptic, a pesticide for wood, leather, and glue preservation, and as an antimildew treatment. Due to its high toxicity, carcinogenic properties, and persistence in the environment $[22,23]$, much attention has been focused on developing cost-effective analytical methods for determining these compounds in environmental samples at low concentration levels. Nowadays, noncovalent molecularly imprinted polymers (MIPs) have increasingly been developed as mimics of natural molecular receptors. However, to the best of our knowledge, there has been no other report of the preparation of 2,4,6-TCP-imprinted microsphere polymers by precipitation polymerization, which could be used as HPLC sorbent for the separation analytes from complex matrices.

To answer the need for fast and straightforward routes to spherical molecularly imprinted polymers, we therefore report the preparation of MIPs selective for 2,4,6-TCP by precipitation polymerization. Factors that might influence the binding characteristics, such as the type and the amount of the functional monomers, the type and the amount of the cross-linkers, the porogen, and polymerization temperature have been investigated and are discussed in detail. The polymers were evaluated in parallel with corresponding irregularly shaped particles prepared from bulk polymer.

\section{Experimental}

\section{Materials}

2,4,6-TCP, 4-vinylpyridine (4-VP), methyacrylic acid (MAA), and ethylene glycol dimethacrylate (EGDMA) were all from Acros Organics; trimethylolpropane trimethacrylate (TRIM) and divinylbenzene (DVB) were from Sigma-Aldrich Chemie. 2,2'-Azobisisobutyronitrile (AIBN) was from Shanghai No.4 Reagent and H.V. Chemical Company (Shanghai China), and acetonitrile was from J. T. Baker (USA). The monomers were purified by standard procedures to remove stabilizers. AIBN was recrystallized from ethanol. Acetic acid and chloroform were purchased from Beijing Chemical Reagent Company (Beijing, China); methanol, ethanol, and toluene were from Beijing Bei Hua Finachemical Company (Beijing, China).

Preparation of bulk and micro/submicrosphere imprinted polymers

For preparation of the imprinted polymers, the template molecule 2,4,6-TCP (1 mmol), the functional monomer MAA or 4-VP, and the cross-linker EGDMA, DVB, or TRIM were dissolved in the porogen [24] following the molar ratios listed in Table 1. After addition of AIBN $(0.5 \mathrm{mmol})$, the solutions were sonicated for $10 \mathrm{~min}$ and deoxygenated with nitrogen for $10 \mathrm{~min}$ and then thermally polymerized at different temperatures for $24 \mathrm{~h}$. The resulting bulk polymers were ground, and then wetsieved/collected with acetone through a $40-\mu \mathrm{m}$ sieve. The fine particles were removed by repetitive sedimentation in acetone. The obtained precipitation polymers (except $11^{\#}$ ) were separated from the reaction medium by centrifugation for $20 \mathrm{~min}$ at 3,500 rpm using an Anke centrifuge (TDL40B, Shanghai, China). All the polymers were washed with acetonitrile/acetic acid $(90: 10, \mathrm{v} / \mathrm{v})$ by Soxhlet extraction until no 2,4,6-TCP was detected in the washing solution 
Table 1 Conditions for the synthesis of polymers with 2,4,6-TCP as the template molecule

\begin{tabular}{|c|c|c|c|c|c|c|}
\hline Number $^{\#}$ & $\begin{array}{l}\text { Functional } \\
\text { monomer }\end{array}$ & $\begin{array}{l}\text { Cross- } \\
\text { linker }\end{array}$ & Porogen & $\begin{array}{l}\text { Amount of } \\
\text { porogen }(\mathrm{mL})\end{array}$ & $\begin{array}{l}\text { Molar ratio (template molecule/functional } \\
\text { monomer/cross-linker) }\end{array}$ & $\begin{array}{l}\text { Temperature } \\
\left({ }^{\circ} \mathrm{C}\right)\end{array}$ \\
\hline 1 & MAA & EGDMA & Toluene & 60 & $1: 4: 20$ & 60 \\
\hline 2 & MAA & EGDMA & $\begin{array}{l}\text { Toluene/acetonitrile } \\
(1: 3, \mathrm{v} / \mathrm{v})\end{array}$ & 60 & $1: 4: 20$ & 60 \\
\hline 3 & MAA & DVB & $\begin{array}{l}\text { Toluene/acetonitrile } \\
(1: 3, \mathrm{v} / \mathrm{v})\end{array}$ & 60 & $1: 4: 20$ & 60 \\
\hline 4 & MAA & DVB & Acetonitrile & 60 & $1: 4: 20$ & 60 \\
\hline 5 & 4-VP & DVB & $\begin{array}{l}\text { Toluene/acetonitrile } \\
(1: 3, \mathrm{v} / \mathrm{v})\end{array}$ & 60 & $1: 4: 20$ & 60 \\
\hline 6 & MAA & EGDMA & Toluene & 60 & $1: 4: 20$ & 50 \\
\hline 7 & MAA & EGDMA & Toluene & 60 & $1: 4: 20$ & 70 \\
\hline 8 & MAA & TRIM & $\begin{array}{l}\text { Toluene/acetonitrile } \\
(1: 3, \mathrm{v} / \mathrm{v})\end{array}$ & 60 & $1: 4: 20$ & 60 \\
\hline 9 & MAA & DVB & Toluene & 60 & $1: 4: 20$ & 60 \\
\hline 10 & MAA & DVB & $\begin{array}{l}\text { Toluene/acetonitrile } \\
(1: 3, \mathrm{v} / \mathrm{v})\end{array}$ & 60 & $1: 8: 40$ & 60 \\
\hline 11 & MAA & DVB & Acetonitrile & 6 & $1: 4: 20$ & 60 \\
\hline
\end{tabular}

with the UV-2101PC spectrophotometer at the wavelength of $\lambda_{\max }=294 \mathrm{~nm}$. The particles were dried at $40{ }^{\circ} \mathrm{C}$ for $12 \mathrm{~h}$. As a control, nonimprinted bulk polymers (irregular), microsphere polymers $(3-5 \mu \mathrm{m})$, and submicrosphere polymers $(<1 \mu \mathrm{m})$ were prepared by exactly the same synthetic routes in the absence of the template molecules.

\section{Evaluation of polymers}

To evaluate the polymers, these polymer particles were suspended in water, and the solutions after centrifugation were determined with the UV-2101PC spectrophotometer (Shimadzu Corporation, Japan). A 5.0-mg portion of washed and dried MIPs was added into $5 \mathrm{~mL}$ 2,4,6-TCP solution at different concentrations. The suspensions were then sealed and oscillated for $20 \mathrm{~h}$ at room temperature. After being centrifuged at $3,500 \mathrm{rpm}$ for $20 \mathrm{~min}$, the concentration of free 2,4,6-TCP in the supernatant was detected by measuring the UV absorbance at $\lambda_{\max }=294 \mathrm{~nm}$. The amount of 2,4,6-TCP bound to the polymer was calculated by subtracting the concentration of free 2,4,6TCP from the initial concentration.

The product morphology was observed with scanning electron microscopy (SEM) (S-3000N, Hitachi, Japan) and transmission electron microscopy (TEM) (H-7500, Hitachi, Japan). As part of the morphological characterization, the porosity and surface area of the developed micro/ submicrospheres and bulk polymers were characterized through nitrogen adsorption/desorption analysis using a nitrogen surface area analyzer (ASAP2000, Micromeritics, USA) and Brunauer-Emmett-Teller 20-point isotherm calculations.

\section{FIAD analysis}

The binding properties of the polymer were investigated using Freundlich isotherm affinity distribution (FIAD) analysis, which has been used elsewhere [25] to determine the binding affinity and heterogeneity of MIPs. This model is based on the Freundlich isotherm, Eq. (1), which assumes a power function relationship between the concentration of bound $(B)$ and the free $(F)$ guest molecules. The fitting parameter $m$ is known as the heterogeneity index. The value of $m$ varies from 0 to 1 , with 1 being homogeneous and values approaching 0 being increasingly heterogeneous:

$B=a F^{m}$

The Freundlich model is most easily applied by replotting the experimental binding isotherm in $\log B$ versus $\log F$ format, Eq. (2). The Freundlich model has been shown to be generally applicable to most noncovalently imprinted polymers [26]. A literature survey of noncovalent MIPs, with widely varying templates, polymerization conditions, and binding affinities, found that 11 of 12 were in excellent agreement with the FI $\left(R^{2}>0.95\right)$ [26].

$\log B=m \log F+\log a$

In a typical binding experiment [27], for the batch method, $5.0 \mathrm{mg}$ washed and dried MIP was added into $5 \mathrm{~mL}$ 2,4,6-TCP solution at different concentrations (15$\left.350 \mathrm{mg} \mathrm{L}{ }^{-1}\right)$. The suspensions were then sealed and oscillated for $20 \mathrm{~h}$ at room temperature to ensure equilibration. After being centrifuged at 3,500 rpm for $20 \mathrm{~min}$, the concentration of free 2,4,6-TCP $(F)$ in the supernatant was detected by measuring the UV absorbance at $\lambda_{\max }=$ 
$294 \mathrm{~nm}$. The amount of 2,4,6-TCP bound to the polymer $(B)$ was calculated by subtracting the concentration of free 2,4,6-TCP from the initial concentration. Experimental binding isotherms were analyzed using the FIAD method. The experimentally obtained data were plotted in $\log B$ versus $\log F$ format, and a Freundlich isotherm was fitted to the log plot of the experimental adsorption isotherms.

Absorption kinetics and release experiments on imprinted microspheres

For the dynamic method, $30.0 \mathrm{mg}$ imprinted microsphere polymers $\left(3^{\#}\right)$ was added to $2,4,6$-TCP aqueous solution (200 $\mathrm{mg} \mathrm{L}^{-1}, 30.0 \mathrm{~mL}$ ). The solution was shaken at room temperature. The absorption of the solution at $\lambda_{\max }=$ $294 \mathrm{~nm}$ was determined at $10 \mathrm{~min}, 30 \mathrm{~min}, 2 \mathrm{~h}, 6 \mathrm{~h}$, and $20 \mathrm{~h}$. For the release experiments, $30.0 \mathrm{mg}$ imprinted microsphere polymers $\left(3^{\#}\right)$ was saturated with $30.0 \mathrm{~mL}$ $200 \mathrm{mg} \mathrm{L}^{-1} 2,4,6-\mathrm{TCP}$ aqueous solution for $48 \mathrm{~h}$. Unbound $2,4,6-\mathrm{TCP}$ in the solution was removed by centrifugation at $3,500 \mathrm{rpm}$ for $20 \mathrm{~min}$. The bound polymer particles were then incubated with $20 \mathrm{~mL}$ water and the amount of 2,4,6TCP released from the imprinted polymer matrices was determined with the UV-2401PC.

\section{Results and discussion}

Polymer synthesis and characterization

Nearly monodisperse, spherical particles can be routinely prepared in good yields by precipitation polymerization [28], and it is possible to tune the size and porosity of the particles through control of the polymerization conditions [24]. Ye's group [28] has showed that the yield of bulk polymerization was approximately $40 \%$, while the yield of precipitation polymerization was approximately $85 \%$. In order to facilitate the interpretation of the results reported in this study, the polymerization methods were adapted to achieve suitable conditions aimed at the most objective comparison between the different MIP materials. For instance, the type and ratio of functional monomer to crosslinker used for the preparation of microspheres were similar to the preparation of bulk polymers. The equilibrium binding analysis was carried out as described in FIAD analysis.

\section{Selection of functional monomer}

It is of obvious importance that the functional monomers strongly interact with the template prior to polymerization, since the solution structure of the resulting assemblies presumably defines the subsequently formed binding sites. By stabilizing the monomer-template assemblies, it is possible to increase the number of imprinted sites. At the same time, the number of nonspecific binding sites will be minimized, since there will be a reduction in the amount of free, nonassociated functional monomer.

For noncovalent imprinting systems, the key interactions include hydrogen bonding and electrostatic interactions. A typical functional monomer for noncovalent imprinting is methyacrylic acid (MAA), whose use was first proposed by Mosbach et al. [29]. The broad applicability of MAA as a functional monomer is related to the fact that the carboxylic acid group serves well as a hydrogen bond and proton donor as well as a hydrogen bond acceptor. In aprotic solvents such as acetonitrile and chloroform, ethyl adenine9-acetate and $N$-Z-L-Glu MIPs were prepared successively with MAA as functional monomer [1, 30]. Often, a large excess of MAA in the synthesis step is required for recognition to be observed and then only in solvents of low to medium polarity and hydrogen bond capacity [31]. The excess of functional monomer results in a portion not being associated with imprinted sites. These sites interact nonselectively with solutes with affinity for templates and limit the degree of separation that can be achieved. Hence MAA, although broadly applicable, is not a universal monomer for the generation of high-affinity sites. 4-Vinylpyridine(4-VP) is particularly well suited for the imprinting of carboxylic acid templates and provides materials with selectivity of the same order as those obtained using MAA for basic templates. However, these polymers are susceptible to oxidative degradation and require special handling [32].

Polymers $3^{\#}$ and $5^{\#}$ were synthesized under the same conditions except for the functional monomers. Although the particle sizes were identical, as shown in Fig. 1a and b, Fig. 2a showed that polymer $3^{\#}$ had better recognition efficiency than that of polymer $5^{\#}$. Theoretically, 2,4,6-TCP is an acidic compound which may easily interact with the basic 4-VP, but the experiments showed the reverse, maybe because MAA has the carboxylic acid group serving well as a hydrogen bond and proton donor as well as a hydrogen bond acceptor in this study, which was agreement with the report [33] that many $\beta$-estradiol-imprinted polymers have been prepared using MAA as functional monomer.

\section{Selection of cross-linker}

Another important factor in molecular imprinting is the type and quantity of the cross-linker, which are both critical for attaining high affinity. In this study, several cross-linking monomers have been evaluated in molecular imprinting, such as ethyleneglycoldimethacrylate (EGDMA), divinylbenzene (DVB), and trimethylolpropane trimethacrylate (TRIM). EGDMA is the most commonly used cross-linker for the methacrylate-based system, primarily because it provides materials with mechanical and thermal stability, 
good wettability in most rebinding media, and rapid mass transfer with good recognition properties [34]. Except for trimethacrylate monomers, such as TRIM, no other crosslinking monomer provides similar recognition properties for such a large variety of templates [32]. TRIM was used to prepare a molecularly imprinted solid-phase extraction (MISPE) sorbent possessing the highest binding selectivity and binding capability for the template tebuconazole [35].
DVB is also an alternative, e.g., Wei et al. [24] prepared a precipitation polymer with high binding selectivity by using DVB.

Polymers $3^{\#}, 2^{\#}$, and $8^{\#}$ were synthesized under the same polymerization conditions except for the type of crosslinkers. Figure 1a,c, and d showed that since there was only considerably weak interaction between MAA and EGDMA (or TRIM), poly(MAA-co-EGDMA) or poly(MAA-co-

Fig. 1 a-k SEM and TEM of imprinted polymers $3^{\#}, 5^{\#}, 2^{\#}$, $8^{\#}, 6^{\#}, 7^{\#}, 1^{\#}, 4^{\#}, 10^{\#}, 9^{\#}$, and $11^{\#}$, respectively
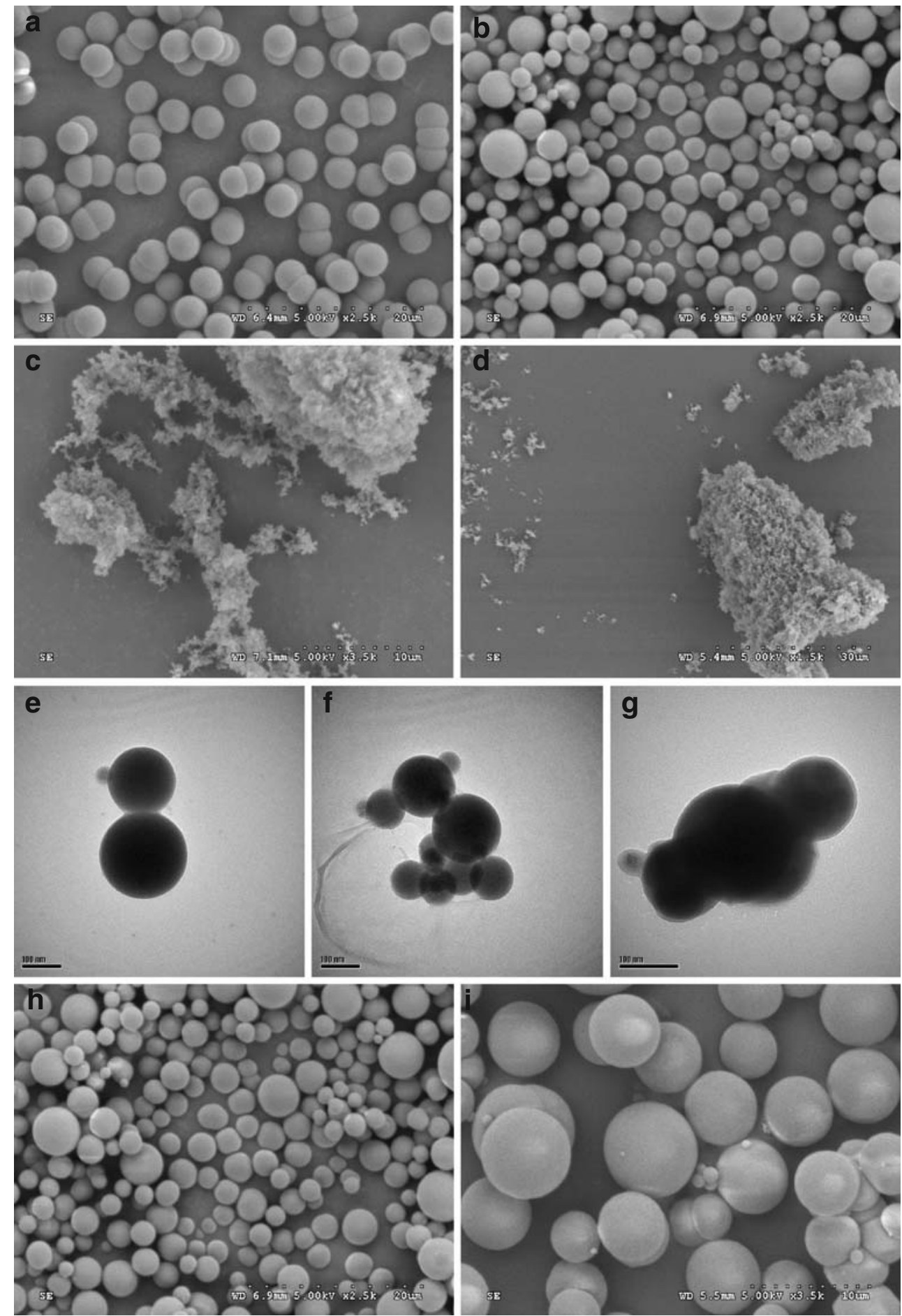
Fig. 1 (continued)
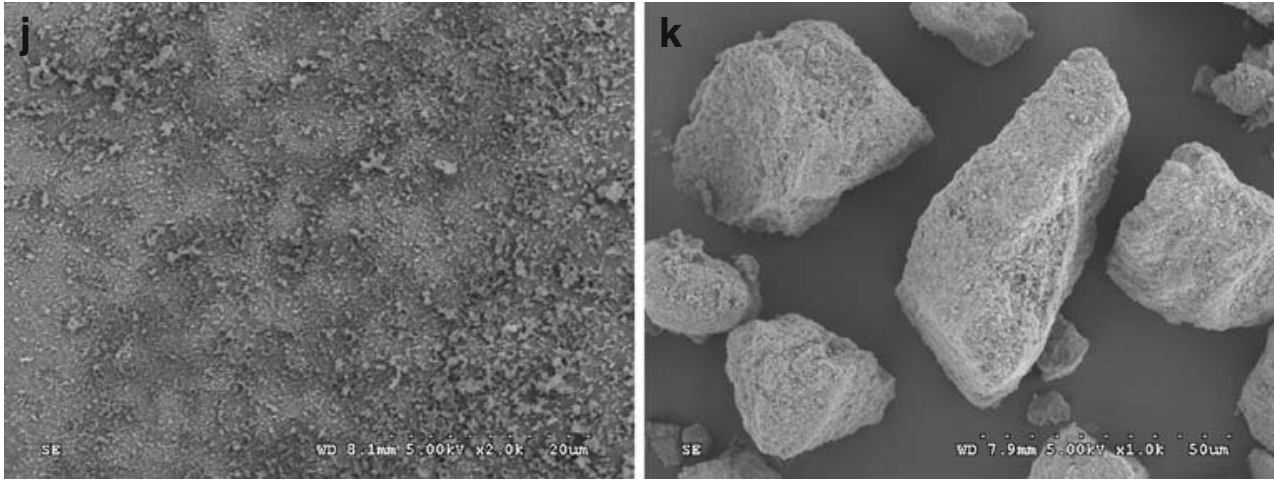

TRIM) tended to form particles with smaller dimensions, while poly(MAA-co-DVB) tended toward particles of larger dimensions if the same ratio of template molecule and monomer to cross-linker was applied (1:4:20). This was because DVB had only one vinyl group to ensure the backbone rigidity. Figure $2 \mathrm{~b}$ and $\mathrm{c}$ showed that polymer $3^{\#}$ had slightly better recognition efficiency than polymers $2^{\#}$ and $8^{\#}$. Therefore, poly(MAA-co-DVB) could be selected for the preparation of microspheres, while poly(MAA-coEGDMA) or poly(MAA-co-TRIM) could be used for the preparation of submicrosphere polymers.

Selection of molar ratio of template molecule and functional monomer to cross-linker

Although the noncovalent imprinting system is simple and convenient, each interaction is weak so that a large excess (at least four times) of the functional monomer should be used. In the traditional preparation method, the molar ratio of template molecule and functional monomer to crosslinker is 1:4:20 [3]. In this study, the molar ratios of 1:4:20 and 1:8:40 were chosen, respectively, to optimize the synthesized polymers.

As presented in Fig. 1a and i, polymers $3^{\#}$ and $10^{\#}$ were microspheres with the same diameter; Fig. $2 d$ showed that the rebinding properties of two kinds of imprinted materials had no significant difference. It might be concluded that the rebinding properties did not depend on the molar ratio, but largely relied on the template-functional monomer interactions, and the cross-linker contributed only in a limited fashion to the specificity for the template. Accordingly, a lower amount of the functional monomer and cross-linker can be used for the preparation the polymer with the spheric particle size by the precipitation method.

\section{Selection of polymerization temperature}

An important part of the optimization process is the stabilization of the monomer-template assemblies which is influenced by the polymerization temperature. In this study, free radical polymerization was chosen using 2,2'azobisisobutyronitrile (AIBN) as initiator. Free radical reactions are little affected by the presence of acids or bases or the polarity of solvents. This allows molecular imprinting to be carried out under a variety of different conditions. The free radical can be generated by heat at different temperatures. This also allows conditions to be chosen where the interactions occurring between monomer and template prior to polymerization are particularly strong. For the common initiator AIBN, the homolysis reaction occurs by heating or irradiation at $345 \mathrm{~nm}$ using a mercury vapor lamp. In the imprinting protocols developed thus far, ca. $1 \%(\mathrm{w} / \mathrm{w})$ AIBN is added based on the total monomer. The thermally initiated polymerizations were carried out at $50{ }^{\circ} \mathrm{C}, 60{ }^{\circ} \mathrm{C}$, and $70{ }^{\circ} \mathrm{C}$, respectively. The influence of temperature on the size of the polymers is presented in Fig. 1e-g.

Figure 2e shows that the polymers prepared at $50{ }^{\circ} \mathrm{C}$ had better binding efficiency, while the binding capacity was lower than the polymers prepared at $60{ }^{\circ} \mathrm{C}$. Worse binding efficiency and binding capacity were found for the polymers prepared at $70{ }^{\circ} \mathrm{C}$. Daniel et al. [36] also studied the influence of the polymerization temperature. The polymers prepared at lower temperature exhibited stronger retentions, although the separation factors were largely similar for the two polymers. In a separate study, it was shown that imprinted polymers prepared at lower temperature exhibited higher separation factors than their counterparts at higher temperature, particularly when evaluating them at lower temperature. If the temperature is too high, the initiation rate is high, too; consequently, a large concentration of reactive centers will be generated leading to early termination of the polymerization and low molecular weights. In contrast, if the rate is too low, the polymerization will not reach completion within a practicable time frame. In the imprinting protocols, the thermally initiated polymerizations were generally carried out at $60^{\circ}$ C. Given the rate constant for decomposition at this temperature $\left(k \approx 1.9 \times 10^{-5} \mathrm{~s}^{-1}\right)$ [32], the rate of radical production should satisfy the above rate criteria. It should 
be noted that this study was also carried out at $70{ }^{\circ} \mathrm{C}$, where the half-life of AIBN is relatively short, in the order of a few hours. The half-life of the initiator responds logarithmically to the temperature; thus the initiator decomposition half life at $60{ }^{\circ} \mathrm{C}$ is more than $10 \mathrm{~h} \mathrm{[32],} \mathrm{whereas} \mathrm{at} 50^{\circ} \mathrm{C}$ it is more than $70 \mathrm{~h}$. Thus, $60^{\circ} \mathrm{C}$ was the optimal temperature for the polymerization.
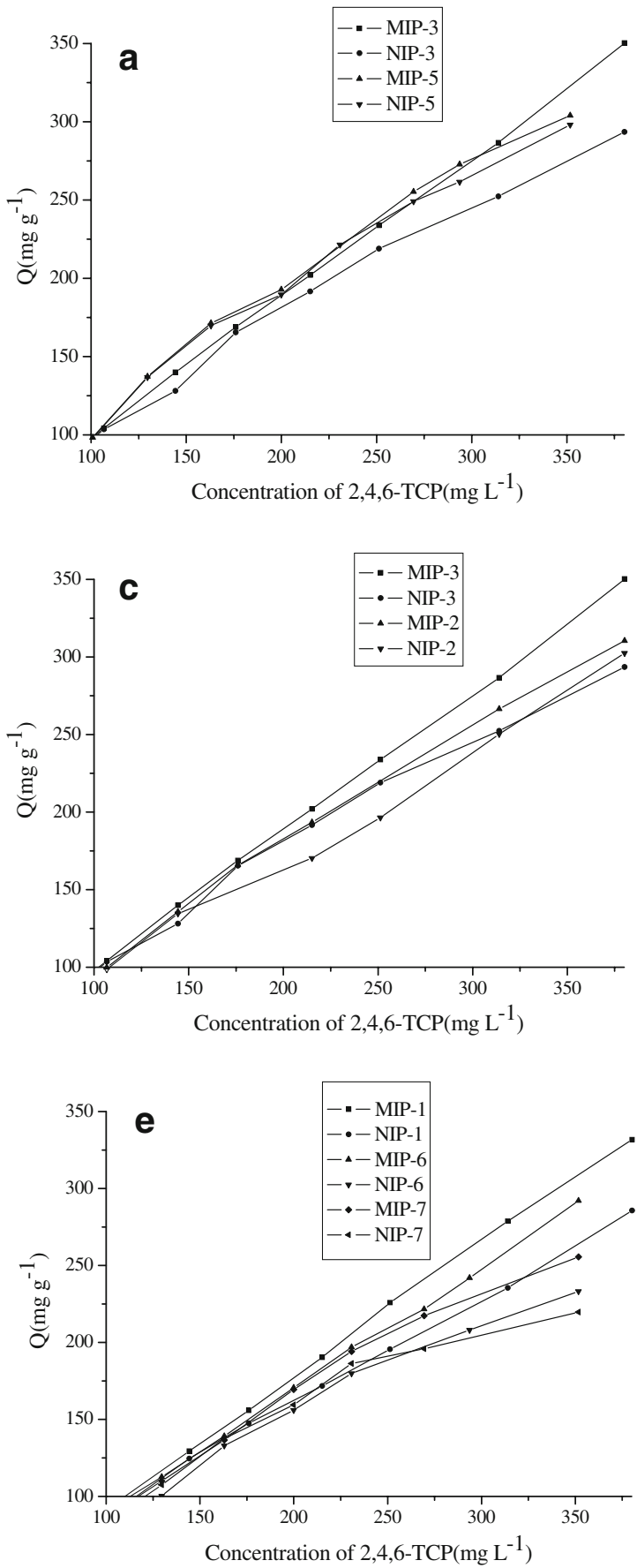

Influence of porogen

In the molecular imprinting, a number of MIPs were prepared using the standard recipe with $83 \%$ cross-linker, but with different kinds of diluents [32]. The different diluents may have different solubility parameters that correlate with the polymer morphology. For instance, in
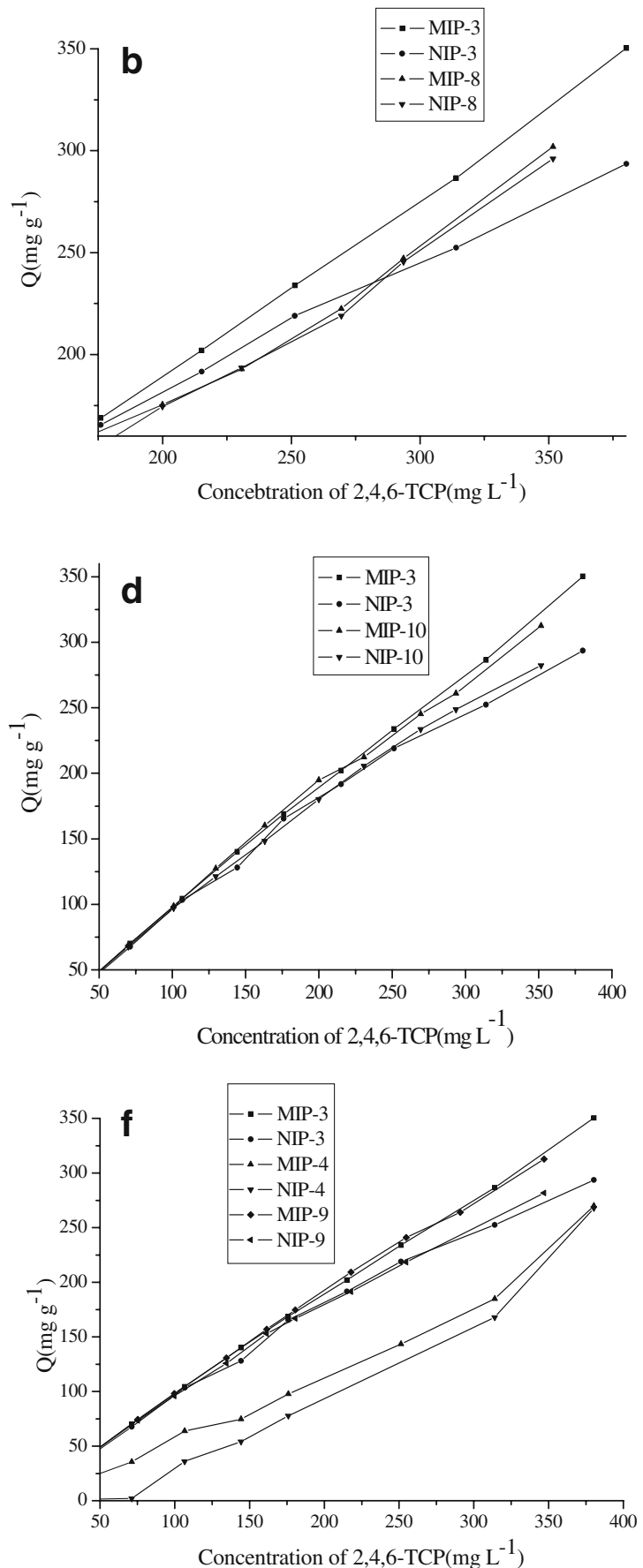

Fig. 2 a-g Binding isotherms of $2,4,6$-TCP-imprinted polymers $3^{\#}$ and $5^{\#} ; 3^{\#}$ and $8^{\#} ; 3^{\#}$ and $2^{\#} ; 3^{\#}$ and $7^{\#} ; 6^{\#}, 7^{\#}$, and $1^{\#} ; 3^{\#}, 4^{\#}$, and $9^{\#}$; and $3^{\#}$ and $11^{\#}$, respectively 


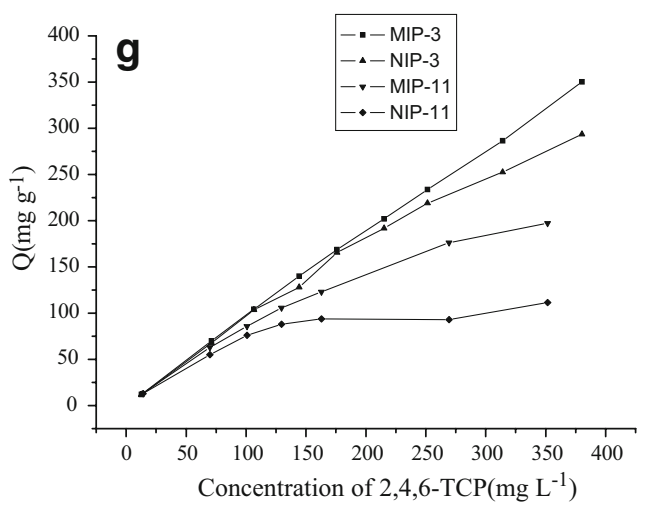

Fig. 2 (continued)

some solvents, agglomeration is promoted, leading to the formation of densely cross-linked microspheres, where the phase separation occurs when the microspheres precipitate. This gives a relatively open pore structure with strong links between the microspheres. Such materials exhibit permanent and low swelling. In the other solvents, intermolecular cross-links are favored and a material is obtained which is built up of loosely linked grains of solvent-swollen gel particles. These materials exhibit low pore volume and high swelling. Therefore, it is very important to decide whether a solvent or a nonsolvent is used as porogen.

In this work, acetonitrile, toluene, and acetonitrile/ toluene $(3: 1, \mathrm{v} / \mathrm{v})$ were used as porogen. As shown in Fig. 1a,h,j, and Fig. 2f, polymers prepared with acetonitrile/ toluene $(3: 1, \mathrm{v} / \mathrm{v})$ had regular particles with bigger size $(3-5 \mu \mathrm{m})$ and better affinity ability, because when DVB is copolymerized under such conditions, phase separation is delayed and the polymer phase is solvated to a reasonable extent, so that materials with well-developed, permanent pore structures and larger average diameters are obtained [37]. In contrast, although polymers prepared with acetonitrile had regular particle size, they had a poor affinity ability. As for polymers prepared with toluene, they had submicrosphere particle size $(400-700 \mathrm{~nm})$ with obvious agglomeration and better affinity ability. Therefore, no correlation was evident between polymer morphology and the rebinding properties of the polymers. Interestingly, this was in disagreement with the finding that better results were obtained using acetonitrile as the diluent in contrast to other systems where solvents of lower polarity (e.g., toluene, dichloromethane, chloroform) gave the best results [32].

Comparison between precipitation polymer and bulk polymer

The bulk materials were crushed, sieved, and sedimented to obtain particles with dimensions $<40 \mu \mathrm{m}$. The crushed bulk polymers provided irregularly shaped particles, as shown in Fig. 1k. Precipitation polymerization resulted in a nearly monodisperse population of spherical particles with diameters in the range of 3-5 $\mu \mathrm{m}$ (Fig. 1a).

Figure $2 \mathrm{~g}$ shows that the bulk polymer had lower binding capacity but better binding efficiency, while the precipitation polymer had higher binding capacity. This indicated that the precipitation polymerization was a straightforward route to spherical molecularly imprinted polymers which could displace the bulk polymerization method. Figure $2 \mathrm{~g}$ also shows that the imprinted factors ( $\left.Q_{\text {maxMIP }} / Q_{\text {maxNIP }}\right)$ for polymers obtained by precipitation polymerization and by bulk polymerization were 1.4 and 1.7 , respectively.

During the development of the molecular imprinting technique, much effort has been directed towards understanding the nature of the imprinting process and optimizing parameters that affect the quality of the imprinted receptor sites. Relatively little effort has been expended in developing polymerization methods that would be amenable to mass production, or in producing polymer particles with improved characteristics for particular end-uses such as chromatography or solid-phase extraction [32]. In this study, the regular particle imprinted polymer could be readily prepared and this polymer could be used as HPLC sorbent. Thus, more experiments should be done to extend its application.

A 500-mg portion of microsphere polymer particles (MIP3, NIP3) was suspended in chloroform and packed into a $50 \times 4.6$-mm stainless steel HPLC column with an airdriven fluid pump (Beifen equipment Company, Beijing, China) at $20 \mathrm{MPa}$ using acetone as the packing solvent. The high-performance liquid chromatograph (HPLC, Shimadzu, Japan) used to determine 2,4,6-TCP consisted of a binary LC-10ADvp pump, a DGU-12A online degasser, a CTO10Avp column oven, an SPD-10Avp UV-Vis detector, and an SCL-10Avp system controller. The injection loop volume was $20 \mu \mathrm{L}$, and the mobile phase consisted of acetonitrile (containing $0.3 \%(\mathrm{v} / \mathrm{v})$ acetic acid). The flow rate of the mobile phase was $0.5 \mathrm{~mL} \mathrm{~min}{ }^{-1}$. The oven temperature was set at $40{ }^{\circ} \mathrm{C}$, and 2,4,6-TCP was detected at $294 \mathrm{~nm}$. An acetone solution was used as the void marker to calculate the capacitor $\left(k^{\prime}\right)$ and the separation factor $(\alpha)$ using basic chromatography theory.

Figure 3 shows that the $k$ ' values for MIP3 and NIP3 were 7.8 and 5.3, respectively, and the $\alpha$ was 1.5 , demonstrating that imprinting effect had been verified and the microsphere polymer could be used as the HPLC sorbent for the separation 2,4,6-TCP from complex matrices.

\section{Porosity studies}

The porosity is determined by the morphology of the material which can be influenced by controlling the degree 


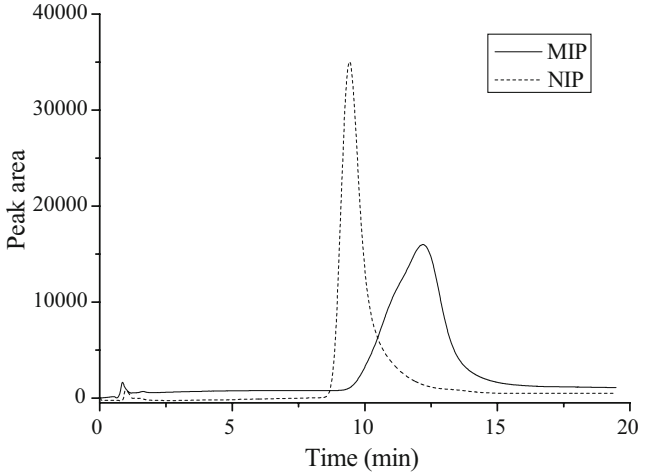

Fig. 3 Chromatogram of 2,4,6-TCP on MIP3 ${ }^{\#}$ and NIP3 ${ }^{\#}$

of cross-linking and the type of inert solvent (porogen) used during polymerization. Table 2 summarizes the pore volume and specific surface area of the polymer particles investigated during this study. It was clearly evident that submicrospheres were characterized by a much smaller pore volume and surface area, as compared to bulk polymers and microspheres. Table 2 also shows that NIP and MIP particles were not significantly different in BET surface and pore size, which proved that the addition of template molecule to the pre-polymerization mixture did not significantly affect the polymer porosity. Therefore, any differential 2,4,6-TCP loadings displayed by the particles would not be caused by the morphological distinction but by the imprinting effect. The total pore volume decreased in the order bulk particles, microspheres, and submicropheres from 0.95 to 0.54 to $0.020 \mathrm{~cm}^{3} \mathrm{~g}^{-1}$, respectively. The differences between the pore volumes and specific surface areas of polymers $11^{\#}, 3^{\#}$, and $1^{\#}$ resulted from the different type and amount of porogen used during the synthesis. This result was in agreement with the findings obtained by Wei et al. [24, 38]. A solvent mixture of toluene/acetonitrile $(1: 3, \mathrm{v} / \mathrm{v})$ could be used as porogen for MIPs synthesized by the precipitation method minimizing the interference of a polar solvent like acetone with hydrogen bonding, thereby facilitating the creation of monodisperse microspheres.

FIAD analysis of microsphere polymers

Microsphere imprinted polymer (polymer $3^{\#}$ ) was used for FIAD experiments because it was to be used as HPLC sorbent
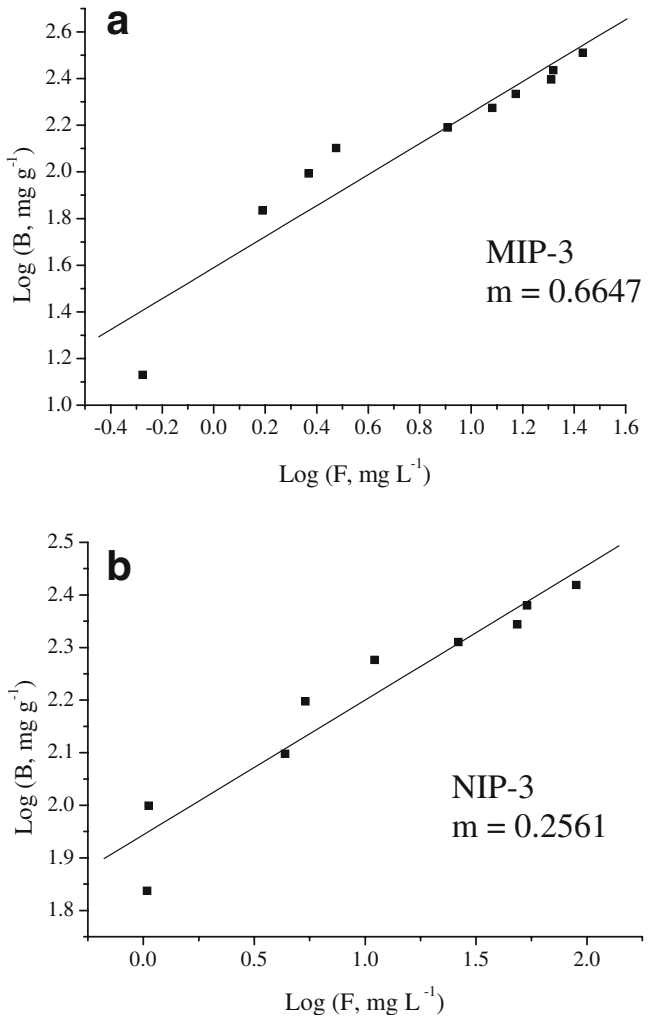

Fig. 4 Freundlich isotherm affinity distribution (FIAD) analysis of the microsphere polymers: a MIP $(m=0.6647)$, b NIP $(m=0.2561)$

in our later experiments. FIAD analysis was carried out as described in the Experimental. As seen in Fig. 4, the heterogeneity index $(m)$ of MIP was 0.6647 , while the heterogeneity index of NIP was only 0.2561 , which was the reason that MIP had better affinity ability than NIP.

Surfaces with recognition abilities can be divided into two general categories: homogeneous and heterogeneous [25]. In homogeneous systems, all the binding sites have the same affinity and selectivity as depicted by the identical depths and shapes of the binding cavities. In contrast, a heterogeneous system contains binding sites of varying affinity and selectivity as denoted by the varying depths and shapes. Various methods have been applied to reduce the heterogeneity in MIPs such as the optimization of the imprinting process or selective chemical modification of the surface. One of the most successful methods was to use stoichiometric or covalent imprinting mechanisms [39].

Table 2 Porosities of molecularly imprinted polymers prepared as bulk polymer, microspheres, and submicrospheres, as determined by BET analysis

\begin{tabular}{lcccccc}
\hline Sample & Microsphere MIP & Microsphere NIP & Bulk MIP & Bulk NIP & Submicrosphere MIP & Submicrosphere NIP \\
\hline Pore volume $\left(\mathrm{cm}^{3} \mathrm{~g}^{-1}\right)$ & 0.542 & 0.309 & 0.950 & 0.424 & 0.020 & 0.012 \\
BET surface area $\left(\mathrm{m}^{2} \mathrm{~g}^{-1}\right)$ & 614.07 & 567.25 & 298.49 & 327.87 & 11.63 & 11.05 \\
Pore size $(\AA)$ & 25.86 & 24.20 & 87.07 & 51.76 & 69.34 & 43.64 \\
\hline
\end{tabular}


This reduces the structural and statistical variability of the key pre-polymerization complex. However, despite the rapid improvement of MIPs, binding site heterogeneity remains an almost inherent property of MIPs. This is particularly true for the noncovalently imprinted polymers, which remain the most common class of MIPs because of their ease of preparation from commercially available monomers [25].

Adsorption kinetics and release experiments on imprinted microspheres

A rebinding kinetics study had been carried out for microsphere polymer $3^{\#}$ particles to determine the rate of the adsorption process, since this would be useful in our later experiments. This is an essential consideration in the actual application of the material in the separation process. As shown in Fig. 5, the MIP based on polymer $3^{\#}$ adsorbed 2,4,6-TCP very quickly in the first $2 \mathrm{~h}$, after which the speed declined rapidly; after $6 \mathrm{~h}$, the MIP no longer adsorbed 2,4,6-TCP. This was the typical kinetics for most rebinding processes. During the initial rebinding process, the presence of a large amount of unoccupied, high-affinity binding sites on the surface of the particles enabled fast adsorption of 2,4,6-TCP molecules. When all the cavities on the surface were occupied, the template molecules should transport to the inner surface of the MIP which would take much more time. When most binding sites were filled up, the rate of adsorption dropped significantly and the 2,4,6-TCP loading was eventually achieved.

The desorption kinetics is also an important consideration in the regeneration of the adsorbent material for repeated use. The kinetics of binding experiments was also studied by release experiments. The polymer materials, which had been equilibrated with 2,4,6-TCP, were incubated with fresh water under shaking to release the re-bound template, and the released amount was temporally monitored. The results are presented in Fig. 6, which shows that

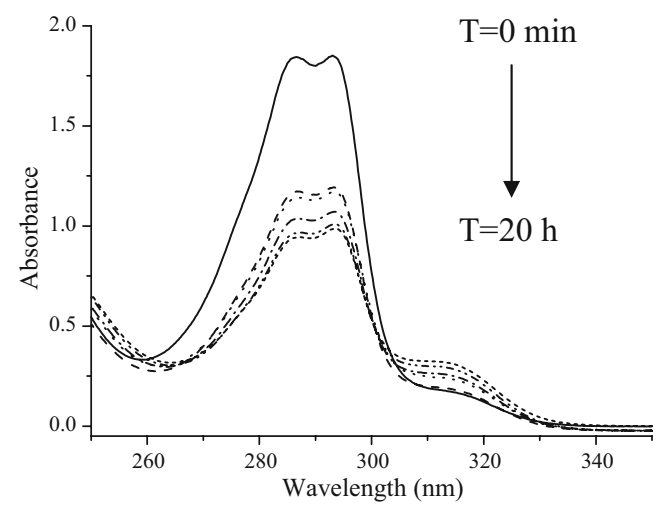

Fig. 5 Adsorption kinetics of MIP (from top to bottom $t=0 \mathrm{~min}$, $10 \mathrm{~min}, 30 \mathrm{~min}, 1 \mathrm{~h}, 2 \mathrm{~h}, 6 \mathrm{~h}, 20 \mathrm{~h}$ )

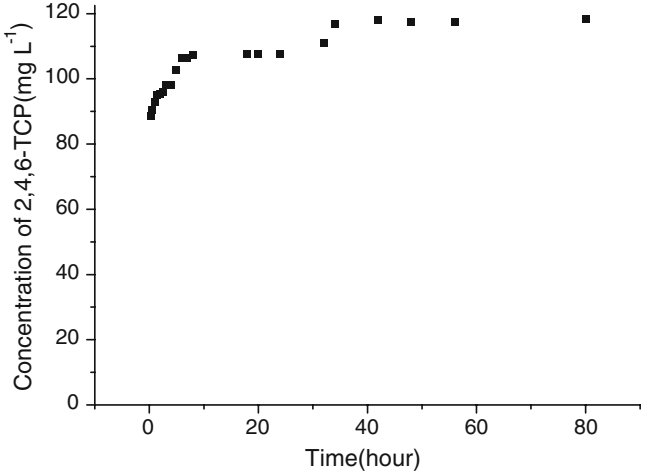

Fig. 6 Release kinetics of MIP

during the first $2 \mathrm{~h}, 70-80 \%$ of $2,4,6-\mathrm{TCP}$ was released from the imprinted particle microspheres, which were the molecules bound with lowest affinity to the polymer matrix. The more tightly bound molecules were released at a significantly longer timescale after continuous incubation, until complete extraction was achieved after $40 \mathrm{~h}$.

\section{Conclusions}

This work detailed the preparation of molecularly imprinted micro- and submicrospheres selective for 2,4,6-TCP by using precipitation polymerization, and these materials were also compared with those obtained by conventional bulk polymerization. The polymer particles prepared by precipitation polymerization exhibited a regular spherical shape for the microsphere with a high degree of monodispersity. Factors that could influence the recognition properties of the polymers were investigated. It was revealed that the imprinted microspheres provide higher affinity to the template in contrast to imprinted polymers prepared by conventional bulk polymerization or with imprinted submicrospheres. Release experiments showed that $80 \%$ of rebound $2,4,6-\mathrm{TCP}$ was released from the imprinted microspheres within the first $2 \mathrm{~h}$, while more tightly bound 2,4,6-TCP molecules were released in the following $40 \mathrm{~h}$. BET analysis indicated that the microspheres prepared by precipitation polymerization provided superior rebinding properties during equilibrium binding in contrast to bulk polymers and submicrospheres. The microand submicrospheres exhibited a comparatively homogenous and narrow distribution of mesopores in contrast to the corresponding bulk polymers. The binding sites distribution appeared more homogeneous as reflected in a heterogeneity index of 0.6647 for the microsphere imprinted polymer.

Acknowledgements This work was supported by the National Natural Science Foundation of China (Grant Nos. 20575073, 20437020 and 20621703), the Major Research Program of the Chinese 
Academy of Sciences (KZCX3-SW-432) and Graduate Innovation Foundation of Chinese Academy of Sciences (No.YXLW-3)

\section{References}

1. Rampey AM, Umpleby RJ, Rushton GT, Iseman JC, Shah RN, Shimizu KD (2004) Anal Chem 76:1123-1133

2. Lin JM, Yamada M (2000) Anal Chem 72:1148-1155

3. Caro E, Marce RM, Cormack PAG, Sherrington DC, Borrull F (2003) J Chromatogr A 995:233-238

4. Caro E, Masque M, Marce RM, Borrull F, Cormack PAG, Sherrington DC (2002) J Chromatogr A 963:169-178

5. Garcia R, Pinel C, Madic C, Lemaire M (1998) Tetrahedron Lett 39:8651-8654

6. Moller K, Nilsson U, Crescenzi C (2001) J Chromatogr A 938:121-130

7. Tsuru N, Kikuchi M, Kawaguchi H, Shiratori S (2006) Thin Solid Films 499:380-385

8. He DY, Zhang ZJ, Zhou HJ, Huang Y (2006) Talanta 69:12151220

9. Lavignac N, Allender CJ, Brain KR (2004) Anal Chim Acta 510:139-145

10. Piletsky SA, Piletska EV, Bossi A, Karim K, Lowe P, Turner APF (2001) Biosens Bioelectron 16:701-707

11. Huang JT, Zheng SH, Zhang JQ (2004) Polymer 45:4349-4354

12. Southard GE, Van Houten KA, Murray GM (2007) Macromolecules 40:1395-1400

13. Oxelbark J, Legido-Quigley C, Aureliano CSA, Titirici MM, Schillinger E, Sellergren B, Courtois J, Irgum K, Dambies L, Cormack PAG, Sherrington DC, De Lorenzi E (2007) J Chromatogr A 1160:215-226

14. Sajonz P, Kele M, Zhong GM, Sellergren B, Guiochon G (1998) J Chromatogr A 810:1-17

15. Kempe H, Kempe M (2006) Anal Chem 78:3659-3666

16. Mayes AG, Mosbach K (1996) Anal Chem 68:3769-3774
17. Kempe H, Kempe M (2004) Macromol Rapid Comm 25:315-320

18. Sambe H, Hoshina K, Moaddel R, Wainer IW, Haginaka J (2006) J Chromatogr A 1134:88-94

19. Prucker O, Ruhe J (1998) Macromolecules 31:602-613

20. de Boer T, Mol R, de Zeeuw RA, de Jong GJ, Sherrington DC, Cormack PAG, Ensing K (2002) Electrophoresis 23:1296-1300

21. Li P, Rong F, Yuan CW (2003) Polym Int 52:1799-1806

22. Pei ZG, Shan XQ, Liu T, Xie YN, Wen B, Zhang S, Khan SU (2007) Environ Pollut 147:764-770

23. Hameed BH (2007) Colloid Surface A 307:45-52

24. Wei ST, Molinelli A, Mizaikoff B (2006) Biosens Bioelectron 21:1943-1951

25. Umpleby RJ, Baxter SC, Rampey AM, Rushton GT, Chen YZ, Shimizu KD (2004) J Chromatogr B 804:141-149

26. Umpleby RJ, Baxter SC, Bode M, Berch JK, Shah RN, Shimizu KD (2001) Anal Chim Acta 435:35-42

27. Xiong Y, Zhou HJ, Zhang ZJ, He DY, He C (2007) Spectrochim Acta A 66:341-346

28. Ye L, Weiss R, Mosbach K (2000) Macromolecules 33:82398245

29. Mosbach K (1994) Trends Biochem Sci 19:9-14

30. Hall AJ, Quaglia M, Manesiotis P, De Lorenzi E, Sellergren B (2006) Anal Chem 78:8362-8367

31. Cheong SH, McNiven S, Rachkov K, Levi R, Yano K, Karube I (1997) Macromolecules 30:1317-1322

32. Sellergren B (2001) Molecularly imprinted polymers, 1st edn. University of Mainz, Germany

33. Sanbe H, Haginaka J (2002) J Pharma Biomed 30:1835-1844

34. Jenkins AL, Uy OM, Murray GM (1999) Anal Chem 71:373-378

35. Hu ML, Jiang M, Wang P, Mei SR, Lin YF, Hu XZ, Shi Y, Lu B, Dai K (2007) Anal Bioanal Chem 387:1007-1016

36. O'Shannessy DJ, Ekberg B, Mosbach K (1989) Anal Biochem 177:144-149

37. Wang JF, Cormack PAG, Sherrington DC, Khoshdel E (2003) Angew Chem Int Ed 42:5336-5338

38. Wei ST, Mizaikoff B (2007) Biosens Bioelectron 23:201-209

39. Wulff G, Knorr K (2001) Bioseparation 10:257-276 